\title{
Uncovering episodic influence of oceans on extreme drought events in Northeast Brazil by ordinal partition network approaches
}

Cite as: Chaos 30, 053104 (2020); https://doi.org/10.1063/5.0004348

Submitted: 11 February 2020 . Accepted: 13 April 2020 . Published Online: 04 May 2020

Huanyu Wu, Yong Zou (D), Lincoln M. Alves (D), Elbert E. N. Macau (D), Gilvan Sampaio, and Jose A. Marengo

\section{COLLECTIONS}

Paper published as part of the special topic on Rare Events in Complex Systems: Understanding and Prediction Note: This article is part of the Focus Issue, Rare Events in Complex Systems: Understanding and Prediciton.
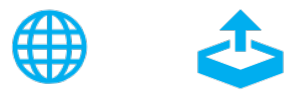

\section{ARTICLES YOU MAY BE INTERESTED IN}

Embedded model discrepancy: A case study of Zika modeling

Chaos: An Interdisciplinary Journal of Nonlinear Science 30, 051103 (2020); https:// doi.org/10.1063/5.0005204

The role of timescale separation in oscillatory ensembles with competitive coupling Chaos: An Interdisciplinary Journal of Nonlinear Science 30, 051101 (2020); https:// doi.org/10.1063/5.0009074

Control of dissipative rogue waves in nonlinear cavity optics: Optical injection and timedelayed feedback

Chaos: An Interdisciplinary Journal of Nonlinear Science 30, 053103 (2020); https:// doi.org/10.1063/5.0003225

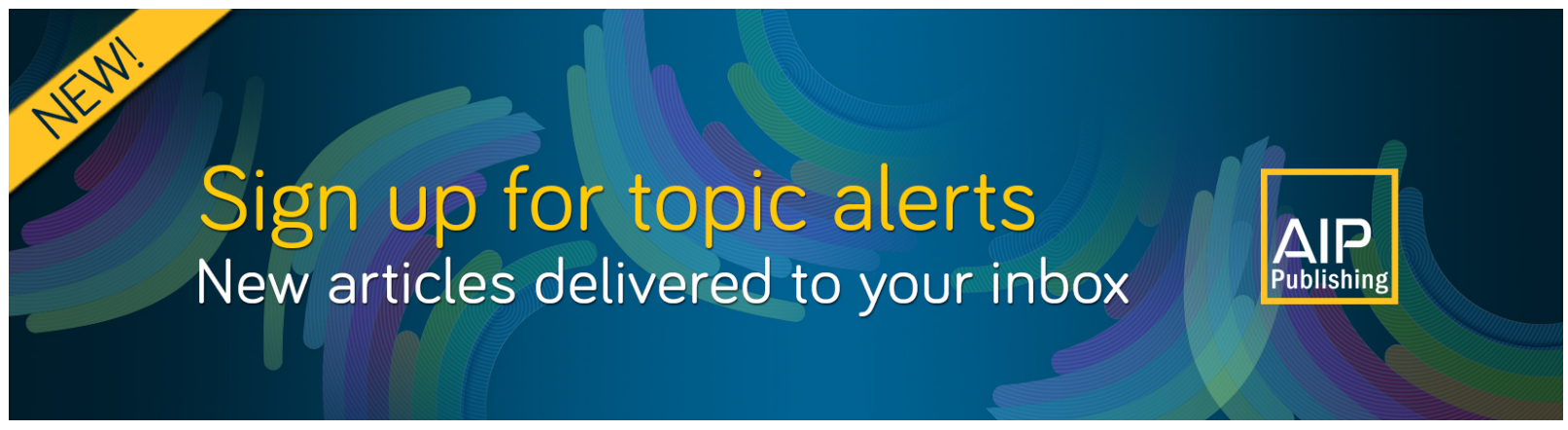




\title{
Uncovering episodic influence of oceans on extreme drought events in Northeast Brazil by ordinal partition network approaches
}

\author{
Cite as: Chaos 30, 053104 (2020); doi: 10.1063/5.0004348 \\ Submitted: 11 February 2020 . Accepted: 13 April 2020 . \\ Published Online: 4 May 2020
}

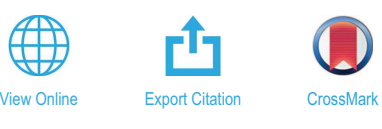

Huanyu Wu, ${ }^{1}$ Yong Zou, ${ }^{1, a)}$ (iD Lincoln M. Alves, ${ }^{2}$ (D) Elbert E. N. Macau, ${ }^{2,3}$ (iD Gilvan Sampaio, ${ }^{4}$ and Jose A. Marengo ${ }^{5}$

\begin{abstract}
AFFILIATIONS
${ }^{1}$ School of Physics and Electronic Science, East China Normal University, Shanghai 200062, China

${ }^{2}$ Instituto Nacional de Pesquisas Espaciais, São José dos Campos 12246-021, São Paulo, Brazil

${ }^{3}$ Federal University of São Paulo, São José dos Campos 12247-014, São Paulo, Brazil

${ }^{4}$ Instituto Nacional de Pesquisas Espaciais, Cachoeira Paulista 12.630-000, São Paulo, Brazil

${ }^{5}$ CEMADEN, Brazilian National Center for Monitoring and Early Warning of Natural Disasters, São José dos Campos 12245-320,

São Paulo, Brazil
\end{abstract}

Note: This article is part of the Focus Issue, Rare Events in Complex Systems: Understanding and Prediciton.

author to whom correspondence should be addressed: yzou@phy.echu.edu.ch

\begin{abstract}
Since 2012, the semiarid region of Northeast Brazil (NEB) has been experiencing a continuous dry condition imposing significant social impacts and economic losses. Characterizing the recent extreme drought events and uncovering the influence from the surrounding oceans remain to be big challenges. The physical mechanisms of extreme drought events in the NEB are due to varying interacting time scales from the surrounding tropical oceans (Pacific and Atlantic). From time series observations, we propose a three-step strategy to establish the episodic coupling directions on intraseasonal time scales from the ocean to the precipitation patterns in the NEB, focusing on the distinctive roles of the oceans during the recent extreme drought events of 2012-2013 and 2015-2016. Our algorithm involves the following: (i) computing drought period length from daily precipitation anomalies to capture extreme drought events; (ii) characterizing the episodic coupling delays from the surrounding oceans to the precipitation by applying the Kullback-Leibler divergence (KLD) of complexity measure, which is based on ordinal partition transition network representation of time series; and (iii) calculating the ratio of high temperature in the ocean during the extreme drought events with proper time lags that are identified by KLD measures. From the viewpoint of climatology, our analysis provides data-based evidence of showing significant influence from the North Atlantic in 2012-2013 to the NEB, but in 2015-2016, the Pacific played a dominant role than that of the Atlantic. The episodic intraseasonal time scale properties are potential for monitoring and forecasting droughts in the NEB in order to propose strategies for drought impacts reduction.
\end{abstract}

Published under license by AIP Publishing. https://doi.org/10.1063/5.0004348

The higher frequencies of El Niño in the Pacific and warmer period of the North Atlantic have a strong influence on rainfall in the region of Northeast Brazil (NEB). In particular, extreme events of droughts are becoming more lasting and intensive in the last 10 years. A drought hazard does not announce its arrival, which can only be discerned in hindsight as the early days of a drought. In the most general sense, drought is usually defined as a deficiency of precipitation over an extended time period of a season or more, resulting in water shortage. Different from this general overview, we propose to compute the expected drought period length to capture the arrival of a drought event, focusing on short temporal properties of droughts. One further challenging task for most nonlinear time series tools is to establish the coupling direction from the surrounding oceans to the precipitation. Here, we focus on a rather novel method of ordinal partition transition network (OPTN)-based complexity measures, which hinges on ordinal symbolic representation of time series. From the viewpoint of methodology, we demonstrate in this work that the OPTN approach is a powerful tool that is capable of disclosing intraseasonal coupling directions from the surrounding oceans to the extreme drought events in the NEB. 


\section{INTRODUCTION}

Located in the extreme Northeast of South America (Fig. 1) and bordered to the north and east by the Tropical Atlantic Ocean, the semiarid region of Northeast Brazil (NEB), with an area of about $1542 \mathrm{~km}^{2}$, is the world's most densely populous dry land region, ${ }^{1}$ with more than 53 million inhabitants, and low economic and social development makes the region one of the world's most vulnerable to extreme climate events. There are several examples of climatic extremes that affected NEB: severe droughts in 1982-1983, 1992-1993, 1997-1998, 2001-2002, 2005, 2007, and 2010 ${ }^{1,2}$ and floods in 1985, 1994, 2004, and 2009. More recently, the drought in the Northeast semiarid region in 2012-2018 shows one of the most significant impacts on population and regional economy in its history. Poor rainfall during the rainy season affected reservoir water availability with severe consequences for urban supply and economic losses in agriculture and livestock. ${ }^{1,3}$

The main characteristic in the NEB is a semiarid climate with a highly spatiotemporal variability of precipitation, that is, some extremely dry years and others extremely rainy years. In general, the NEB climate is a result of the interaction of several physical mechanisms from various time scales, which on seasonal time scales, the intertropical convergence zone (ITCZ), ${ }^{4,5}$ plays an important role in boreal spring (March-April) influenced by the Tropical Atlantic sea surface temperature (SST) anomalies. ${ }^{6,7}$ The abnormal warmer of the SST in the North Atlantic led to an anomalously northward position of the ITCZ, which further causes the shortage of rainfall in the NEB. ${ }^{4}$ In contrast, the presence of positive SST anomalies in the South Atlantic favors the southward displacement of the ITCZ, which in extreme cases cause floods in NEB. In addition, other climatic elements in other regions of the globe, such as El Niño Southern Oscillation (ENSO), have a strong influence on precipitation variability associated with El Niño and La Niña phases, ${ }^{8}$ which often lead to NEB recurrent and profound socioeconomic impacts. Usually, ENSO influences precipitation anomalies over the NEB via changes in the zonally oriented Walter circulation.

It is crucial to emphasize that a full characterization of the teleconnections from the ocean to the precipitation patterns in NEB is

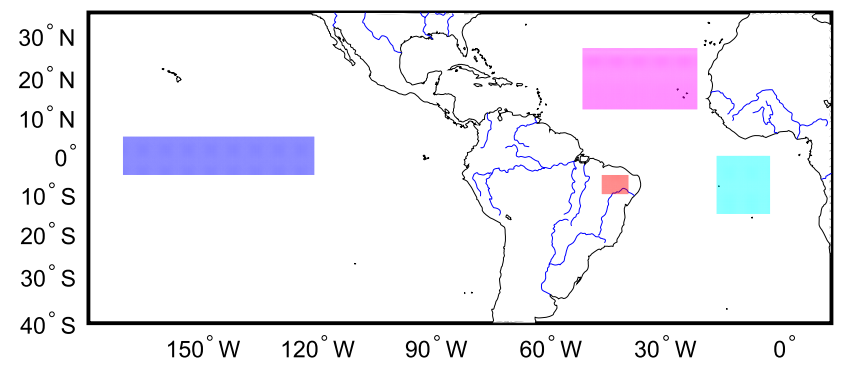

FIG. 1. Geographic locations of interest. The NEB region is between $45^{\circ} \mathrm{W}-38^{\circ} \mathrm{W}, 10^{\circ} \mathrm{S}-5^{\circ} \mathrm{S}$. The Pacific region is represented only by Niño 3.4 between $170^{\circ} \mathrm{W}-120^{\circ} \mathrm{W}, 5^{\circ} \mathrm{S}-5^{\circ} \mathrm{N}$, while Niño 3 and Central and Eastern Pacific are not shown. The Tropical North Atlantic (NA) lies between $50^{\circ} \mathrm{W}-20^{\circ} \mathrm{W}$, $12^{\circ} \mathrm{N}-27^{\circ} \mathrm{N}$, and Tropical South Atlantic (SA) lies between $15^{\circ} \mathrm{W}-0^{\circ} \mathrm{E}, 15^{\circ} \mathrm{S}-0^{\circ} \mathrm{N}$. The north-south difference (NA minus SA across the equator) is a measure of the tropical Atlantic sea surface temperature dipole. still a big challenge since the coupling relationships from tropical Pacific and Atlantic Oceans show considerable variability throughout the rainy season. ${ }^{10-12}$ Observational and theoretical evidence, as well as climate model results, point out that oceanic and atmospheric conditions over the Atlantic and Pacific Tropical basin are determining factors in interannual and decadal climate variability over NEB. ${ }^{6}$ It is hypothesized that the tropical Atlantic and tropical Pacific play distinctive roles in different time scales influencing the precipitation patterns. ${ }^{12}$ In recent years, the most widely accepted interpretation is that tropical Atlantic SST variability was indeed a determinant of NEB rainfall anomalies, while ENSO may at times reinforce them and sometimes weaken them. Despite this evidence, some studies consider that the relationship between ENSO and NEB is not straightforward, and therefore, ENSO teleconnections play an important role in climate variability of the Tropical Atlantic, which, in turn, affects the NEB climate. ${ }^{7,13}$ In fact, only part of drought events in the NEB has been attributed to ENSO, while other events are due to the tropical South Atlantic SST variability., ${ }^{1,14}$ Furthermore, Madden-Julian Oscillation (MJO) originating from the western Pacific is likely to be one of indirect effects changing the short temporal scale of intraseasonal rainfall variability in the South America.

There are a large number of regional studies that used different approaches to analyze the influence of the ENSO and Tropical Atlantic SST on the interannual variability of precipitation over NEB. ${ }^{6,10}$ However, proper statistical tests of the influence mechanisms from the surrounding oceans remain to be an unresolved issue. In particular, it is still one of the big challenges to assess time delays and to quantify the strength of an interacting mechanism because of a strong dependence on serial autocorrelation, ${ }^{16,17}$ which may be resolved by graphical models and well-adapted hypothesis tests. ${ }^{18}$ Recently, complex network approaches have demonstrated to be successful in revealing the global coupling patterns of extreme rainfall events. ${ }^{19}$ Different types of Eastern Pacific and Central Pacific El Niño and La Niña and their distinctive influences on the global climate have been well identified by network-based indices.

Besides these recent developments, time series network approaches provide interdisciplinary platforms for nonlinear time series by means of complex networks. ${ }^{21}$ Based on this consideration, we aim at advancing the understanding of the temporal variability of precipitation over NEB at a regional level, with emphasis on the response of rainfall to SST anomalies of the Equatorial Pacific and Tropical Atlantic oceans, in particular, during the recent decade of drought events. More specifically, we identify episodic intraseasonal coupling delays from the ocean to the precipitation patterns in the NEB using recent time series network approaches.

The outline of this paper is as follows: In Sec. II, we first provide an introduction on datasets that are used in this work. In Sec. III, we illustrate the main steps that are used to characterize the extreme drought events in NEB, including the computation of drought period length, the ordinal partition transition network, the Kullback-Leibler divergence measure, and the hypothesis test. We summarize the main results in Sec. IV, discussing the distinctive roles of Pacific and Atlantic oceans on the drought events in the last 10 years. Finally, our main conclusions are summarized in Sec. V. 


\section{DATA}

We use data of Climate Prediction Center (CPC) gauge-based analysis of global unified daily precipitation for a period of 40 years from 1979 to 2018 with $0.5^{\circ}$ latitude $0.5^{\circ}$ longitude horizontal resolution..$^{22}$ In the $\mathrm{CPC}$, a suite of unified precipitation products are created with consistent quality improvement, which are done by combining all sources of information available at the CPC, by taking advantage of the optimal interpolation and objective analysis techniques. ${ }^{23}$ The sea surface temperature (SST) is evaluated using NOAA of $0.25^{\circ}$ latitude $\times 0.25^{\circ}$ longitude high-resolution data products. ${ }^{24}$

Figure 1 shows the geographic location of the domains. In the Tropical Atlantic Ocean, we selected two areas that represent the Atlantic dipole and reflect the relevance to climate variability of NEB rainfall. ${ }^{6,25}$ In addition, smaller areas in the tropical Atlantic are used because they are the centers with the largest SST anomalies. These Atlantic areas are further east, which should be independent of the ENSO ${ }^{25}$ Moreover, we calculate the north-south Atlantic SST difference to capture the asymmetric SST anomaly pattern about the equator which has been referred to as an SST dipole mode, ${ }^{25}$ showing interannual climate variations over NEB. By the same time, we study the influence of different Pacific areas of Niño 3.4, Niño 3.0, the Central Pacific, and the Eastern Pacific over the precipitation in the NEB, as that has been reported in Refs. 26-28. Note that the Eastern Pacific corresponds to most of Niño 3.0 region, while the
Central Pacific is often defined as Niño 4.0. Some of the droughts in NEB are related to the Eastern Pacific, and it is also most likely a combination of Eastern Pacific and warm tropical Atlantic SST during some drought years in the NEB. ${ }^{26}$ In our work, we obtain consistent results for all Pacific areas, and hence, we only briefly summarize the results for the Central and Eastern Pacific. For each domain, we calculate the spatial average of the variable of interest and calculate the daily anomalies relative to a base period of 1980-2005. This choice of climatology window helps to minimize the long-term trend effects. Additionally, we focus on some specific features for the recent continuous drought events from 2009 to 2018 .

\section{METHODS}

There are three steps in our analysis to assess coupling from the surrounding oceans to the precipitation patterns in the NEB, focusing on the recent increasing trend of the drought events: (i) computation of drought period length from the precipitation anomaly series, (ii) statistically establishing the coupling delays from the ocean to the precipitation by means of time series network approaches, and (iii) assessing the properties of SST during the drought events with proper delays that are identified in step (ii). These three steps are illustrated in Fig. 2, which will be further discussed below.



FIG. 2. The calculation of drought period length for the period 2010-2013. (a) Example precipitation anomaly time series, (b) is the corresponding drought period length series, and (c) SST in the Niño3.4 region and time windows of SSTs larger than $0.5^{\circ} \mathrm{C}$ are highlighted. 


\section{A. Drought period length and drought events}

Drought is a natural hazard and it does not announce its arrival, which can only be discerned in hindsight as the early days of a drought. Hence, it is a slow onset event. When characterizing the arrival of a drought event, we recently proposed to compute the expected drought period length (DPL) to quantify the dry conditions, which have shown the power in capturing the fundamental properties in both the Amazon region ${ }^{29}$ and the exceptional drought year 2014 in São Paulo of the Southeast Brazil region. ${ }^{30}$ More specifically, given the daily precipitation anomaly series $\operatorname{PREC}(t)$, we compute the expected drought period length $\operatorname{DPL}(t)$ that characterizes the waiting time from a given day to the next first non-negative rainfall anomaly as follows:

$$
\operatorname{DPL}(t)=\min \{\tau: \operatorname{PREC}(t+\tau) \geq 0, \tau \in[0, \infty)\} .
$$

The DPL $(t)$ captures the expecting time when anomaly series goes from negative to positive, characterizing the expectation to have an excess rain anomaly. In addition, from the viewpoint of climatology, we also suggest to compute the DPL based on five-day averaged precipitation anomalies, which may reduce the noise effects of daily temporal resolution series. We show an example of computing DPL series from the precipitation anomalies of the period 2010-2013, as illustrated in Fig. 2.

Drought and its effects vary from region to region. In a tropical climate like NEB, a week without rain might be considered a dry spell that could evolve to drought if it becomes longer. In the most general sense, drought is usually defined as a deficiency of precipitation over an extended time period of a season or more, resulting in water shortage. Differently from this general overview, we characterize short temporal properties of droughts following our previous work. ${ }^{29,30}$ More specifically, from the daily DPL series, we define a drought event if the corresponding DPL value is larger than a threshold of two weeks (14 days), which may largely capture the slow arrival time of a drought event. The empirical choice of this threshold is suggested by the mean dry spell period length which is about one week..$^{30}$ Therefore, drought events as defined in this work capture the intraseasonal precipitation variabilities. More drought events are identified if a smaller threshold value is used, which, however, do not change the following results significantly. Note that consistent results have been obtained when different thresholds are used.

\section{B. Coupling delays identified by Kullback-Leibler divergence}

The estimation of coupling direction from the surrounding oceans to the NEB has been obtained by a time series network approach, which has been recently proposed in Refs. 31-33. Here, we present a brief introduction of this algorithm and the details of a general overview of complex network approaches to nonlinear time series analysis have been reviewed in Refs. 21 and 33. More specifically, we focus on ordinal partition transition network (OPTN)-based complexity measures that have been proposed to infer coupling delays from time series. ${ }^{33}$ This method hinges on ordinal symbolic representation of time series. ${ }^{33}$ We note that a weak stationarity should be assumed before the following method is applied. This is physically meaningful since we focus on the intraseasonal time scales, while the ENSO shows on average large time scales of 2-7 years.

Starting from time series of SST as $X=\left\{x_{t}\right\}_{t=1}^{N}$, we first embed time series in phase space using delay embedding, ${ }^{21,31}$ namely, $\vec{x}_{t}=\left[x_{t}, x_{t+\tau_{d}}, \ldots, x_{t+(D-1) \tau_{d}}\right]$ with embedding parameters of dimension $D$ and delay $\tau_{d}$. Then, we use the rank order to represent the embedded vector $\vec{x}_{t}$. Here, the number of unique patterns is decided by dimension $D$ and there are at most $D$ ! different patterns, which are denoted as $\pi_{1}, \pi_{2}, \ldots, \pi_{D !}$. Therefore, the corresponding symbolic series $\left\{\pi_{i}^{X}\right\}_{t=1}^{N}$ is obtained by considering the ordinal pattern of each vector $\vec{x}_{t}$. Time series generated by deterministic dynamical systems often yield an uneven distribution of patterns $p\left(\pi_{i}\right)$, which shows distinctive properties compared to stochastic processes. In this line of research, symbolic methods have been well developed to characterize the underlying dynamical system. ${ }^{34,35}$ The choice of the embedding parameters $\tau_{d}$ and $D$ is often based on the first root of the autocorrelation function of the time series and the false nearest neighbor method, respectively. ${ }^{31}$ In this work, we choose $\tau_{d}=1$ and $D=2$. The following results have been verified by $D=3$. For the precipitation anomaly series $Y=\left\{y_{t}\right\}_{t=1}^{N}$, we obtain its associated ordinal pattern series $\left\{\pi_{j}^{Y}\right\}_{t=1}^{N}$.

The coupling direction from $X \rightarrow Y$ (i.e., SST $\rightarrow$ precipitation) is captured by computing the time-lagged co-occurrence frequencies of ordinal pattern series $\pi_{i}^{X}$ and $\pi_{j}^{Y}$, which was proposed in Ref. 33. More specifically, for $X$ exhibiting an ordinal pattern $\pi_{i}$ at some time $t$, we compute the time-lagged co-occurrence frequencies with all ordinal patterns of $Y$ to be observed at time $t+\tau$, i.e., $p\left(\pi_{j}^{Y}(\tau) \mid \pi_{i}^{X}\right)$. With the null hypothesis that $Y$ is independent of $X$, the co-occurrence frequencies $p\left(\pi_{j}^{Y} \mid \pi_{i}^{X}\right)$ and the marginal distribution $p\left(\pi_{j}^{Y}\right)$ are the same. On the other hand, the difference between these two distributions suggests the coupling. Therefore, we first compute the pattern-wise Kullback-Leibler divergence (KLD) between $p\left(\pi_{j}^{Y} \mid \pi_{i}^{X}\right)$ and $p\left(\pi_{j}^{Y}\right)$ in the following way:

$$
\operatorname{KLD}^{\pi_{i}}(\tau)=\sum_{j=1}^{D !} p\left(\pi_{j}^{Y}(\tau) \mid \pi_{i}^{X}\right) \log _{2} \frac{p\left(\pi_{j}^{Y}(\tau) \mid \pi_{i}^{X}\right)}{p\left(\pi_{j}^{Y}\right)} .
$$

Then, the global KLD complexity measure is therefore calculated as

$$
\operatorname{KLD}(\tau)=\sum_{i=1}^{D !} p\left(\pi_{i}^{X}\right) \operatorname{KLD}^{\pi_{i}}(\tau)
$$

where the summation runs over all patterns $\pi_{i}^{X}$ of $X .^{33}$ The KLD value vanishes if and only if $X$ and $Y$ are independent, while any positive value suggests a possible directed influence from $X$ to $Y$. Furthermore, $\tau=0$ corresponds to the case of simultaneous cooccurrence of ordinal patterns in both sequences, while nonzero lags of $\tau$ capture the case that the driven process $Y$ responds to the driving $X$ with proper delays.

\section{Hypothesis test}

Due to the finite length of time series and possible noise effect, a statistical significance test has to be properly evaluated how likely the causal link from the ocean to the precipitation arise by chance. 
(a)

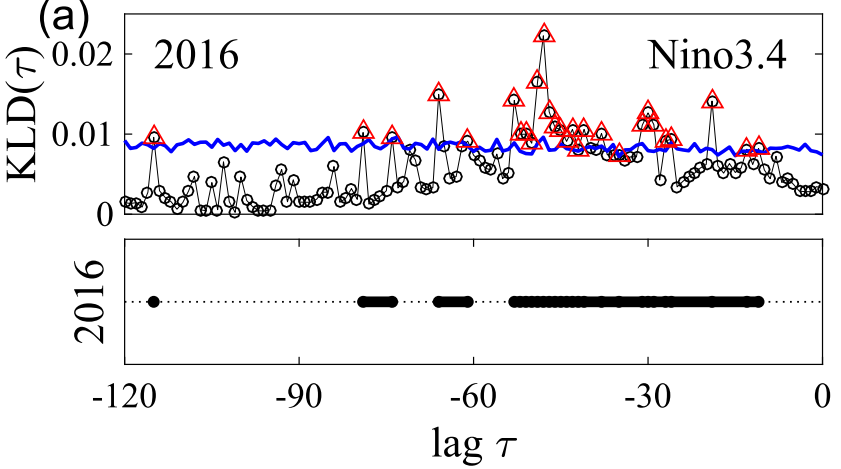

(c)

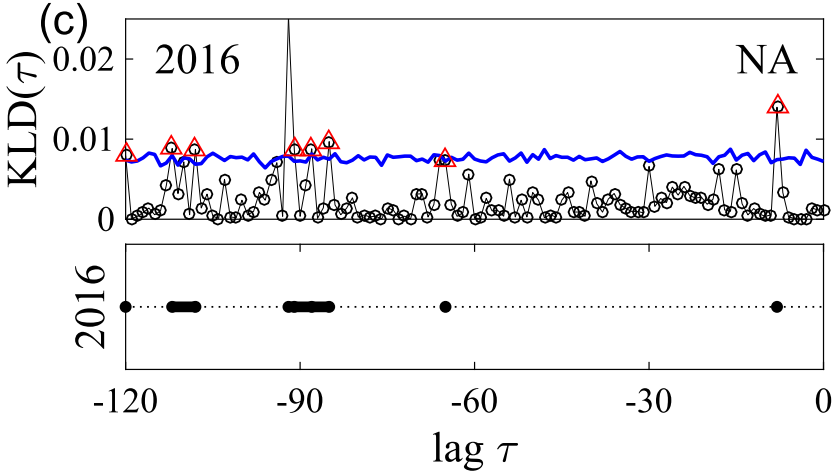

(b)
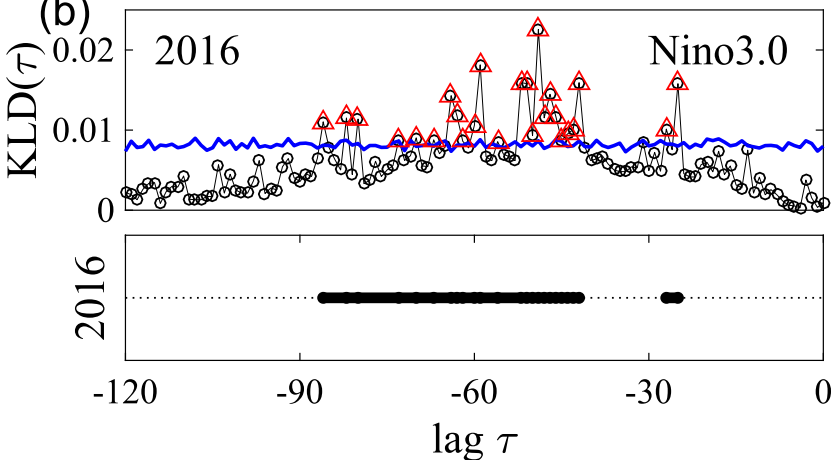

(d)

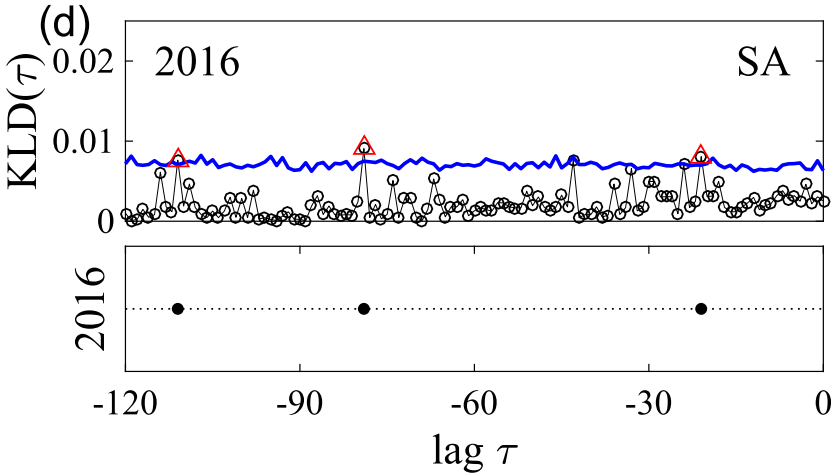

FIG. 3. Kullback-Leibler divergence measure vs time lag for the year 2016 . The solid (blue) line is the threshold value obtained from the $95 \%$ of surrogate sample distribution with size $N_{s}=1000$. The diagram highlights the time lags that cross the significance level. (a) Niño 3.4, (b) Niño 3.0, (c) North Atlantic, and (d) South Atlantic.

Here, the null hypothesis is that the two variables of $X$ and $Y$ (SST and precipitation) are statistically independent. The null hypothesis test is achieved by using a procedure of shuffled surrogate, which generates $N_{s}$ copies of the original variables with the temporal order of the elements randomly shuffled. Here, we generate surrogates either by phase randomizations ${ }^{36}$ or by order pattern transition randomizations, which yield qualitative the same results. The causal interactions of time lags $\tau$ are determined by comparing the KLD of the original pair of time series with a confidence level, which is set at $95 \%$ percentile of the distribution of KLD values calculated from the $N_{s}=1000$ surrogates.

Figure 3 shows the hypothesis test procedure for uncovering nonstationary coupling by KLD. We use the period of 2016 as an example to show the methodology in obtaining the lag information from the surrounding ocean areas. Following similar ideas as in Ref. 17, we consider the period of each year (i.e., 365 sample points). The possible coupling relation is examined by shifting the SST series back in time, from zero lag up to the maximum of four months ( -120 days), which allows one to capture the interaction within the period of interest.

In the upper panel of each subfigure, the black circle-dotted line shows the KLD values against the time lag between the SST and precipitation, and the blue line is the threshold value of $95 \%$ significance level. Whenever the KLD values are larger than its respective threshold, we reject the null hypothesis that the two variables are independent. In other words, those crossings represent significant coupling delays from the ocean to the precipitation patterns, which are further highlighted in the lower panel.

Furthermore, as suggested in Ref. 17, continuous crossings within one week time window are regarded as substantial lag information, which, therefore, avoid isolated crossings. For instance, an isolated crossing at -117 is neglected. More specifically, in the year 2016, we identify 2-3 months coupling lag (at lag positions of -85 , -67 , and -55 ) from the Niño 3.4 to the precipitation in the NEB [Fig. 3(a)]. Although with a slightly different geolocation, it suggests a consistent lag of 3 months from the Niño 3.0 region [Fig. 3(b)]. In the north Atlantic, lags slightly larger than 3 months are identified [i.e., -110 and -95 in Fig. 3(c)]. However, no significant coupling lags have been identified in the south Atlantic area [Fig. 3(d)]. Interestingly, we have obtained qualitatively the same lag information for the Central Pacific as Niño 3.4, while the Eastern Pacific shows the same lags as Niño 3.0.

We simplify the notation for positions of negative lag values by days back in time when discussing about the nonstationary coupling lag information in Sec. IV B. For instance, lag position of -85 is referred to as 85 days back in time from 1st January.

\section{Correlations with SST}

With the time lag information as identified by KLD measure, we look back how the SST of the surrounding oceans behave during the drought events as defined by DPL [as shown in Fig. 2(c)]. 
Starting from a time window $\left[t_{i}, t_{j}\right]$, that is, identified as a drought event by DPL, we study the frequency of extreme high SST in the respective lagged window $\left[t_{i}+\tau, t_{j}\right]$, where $\tau$ is the lags chosen by KLD. Taking the SST of Niño 3.4 as an example, the anomalously warming frequency in the lagged window is captured by computing the ratio as

$$
\text { Ratio }=\frac{\#\left\{\left[t_{i}+\tau, t_{j}\right] \mid \mathrm{SST}>0.5^{\circ} C\right\}}{\#\left\{t_{i}+\tau, t_{j}\right\}},
$$

where $0.5^{\circ} \mathrm{C}$ is a threshold that is often used to define an El Niño event. ${ }^{37}$ For a year of several drought events, the above ratio is an average over the same number of lagged windows. The same calculations have been performed for both the tropical North and South Atlantic oceans, but with a temperature threshold $0.6{ }^{\circ} \mathrm{C}$ since the Atlantic is normally warmer than the Pacific.

In order to focus more on the drought events during the austral summer and fall (wet) seasons, we further restrict the drought events in time windows of February, March, April, and May (FMAM) that represent the peak of the rainy seasons.

\section{RESULTS}

\section{A. General behavior of DPL}

Let us start by showing the general behavior of drought period length for the entire period of 40 years from 1979 to 2018 . Figure 4(a) shows the frequencies of negative precipitation anomaly series of each year. The years of high frequencies have been consistently identified with observations. ${ }^{1,38}$ More specifically, 1979-1983, 1987, 1990-1994, 1997-1999, 2001-2005, 2007, 2010 and 2012-2018 present high frequencies of negative anomalies. From this result, it visually suggests an increasing trend of frequency of larger than 0.5 starting from 2009 till the present 2018, which agrees with the results of Ref. 1.
Due to the possible daily fluctuations in the anomaly series, we calculate the anomaly by a five-day mean of the precipitation. Then, we compute DPL and the general behavior of the frequencies of DPL values larger than two weeks is shown in Fig. 4(b), which show qualitative the same results as in Fig. 4(a).

Particularly, the drought events (from 2009 to 2018) as defined by DPL are further summarized in Table I. It shows much extended long drought periods in 2012-2013 and 2015-2016. These were the worst years in terms of the drought impacts on vegetation, as shown in Ref. 1.

\section{B. Episodic coupling lags}

Nonstationary coupling directions from the surrounding oceans to the precipitation patterns of NEB are identified by $\operatorname{KLD}(\tau)$, which are shown in Fig. 5. These results are further summarized in Table II. Due to the recent increasing trend of drought conditions, we show significant coupling delays for each years separately from 2009 to 2018. Each of these years is referred to as the period of interest (POI) spanning from 1st January until 31st December. This civil year calendar helps to standardize the climatological interpretations. In addition, the time window of the possible driver of SST in the oceans moves continuously back in time regarding to the POI. ${ }^{17}$ In addition, we summarize the results focusing on 2009-2010, 2012-2013, and 2015-2016. The other years will be briefly mentioned when necessary.

Pacific Ocean: Four slightly different regions of the Pacific Ocean show qualitatively the same results for the coupling delays. In particular, time lags of 2-3 months have been identified from the Niño 3.4 and Central Pacific for the years 2009, 2011-2013, 2014, and 2016. This lag is increased up to 4 months for 2015 and 2017. No lags are identified for 2010. The regions of Niño 3.0 and Eastern Pacific are closer to the South America; thus, the lags are shorter than that of Niño 3.4. Specifically, one month delay has been
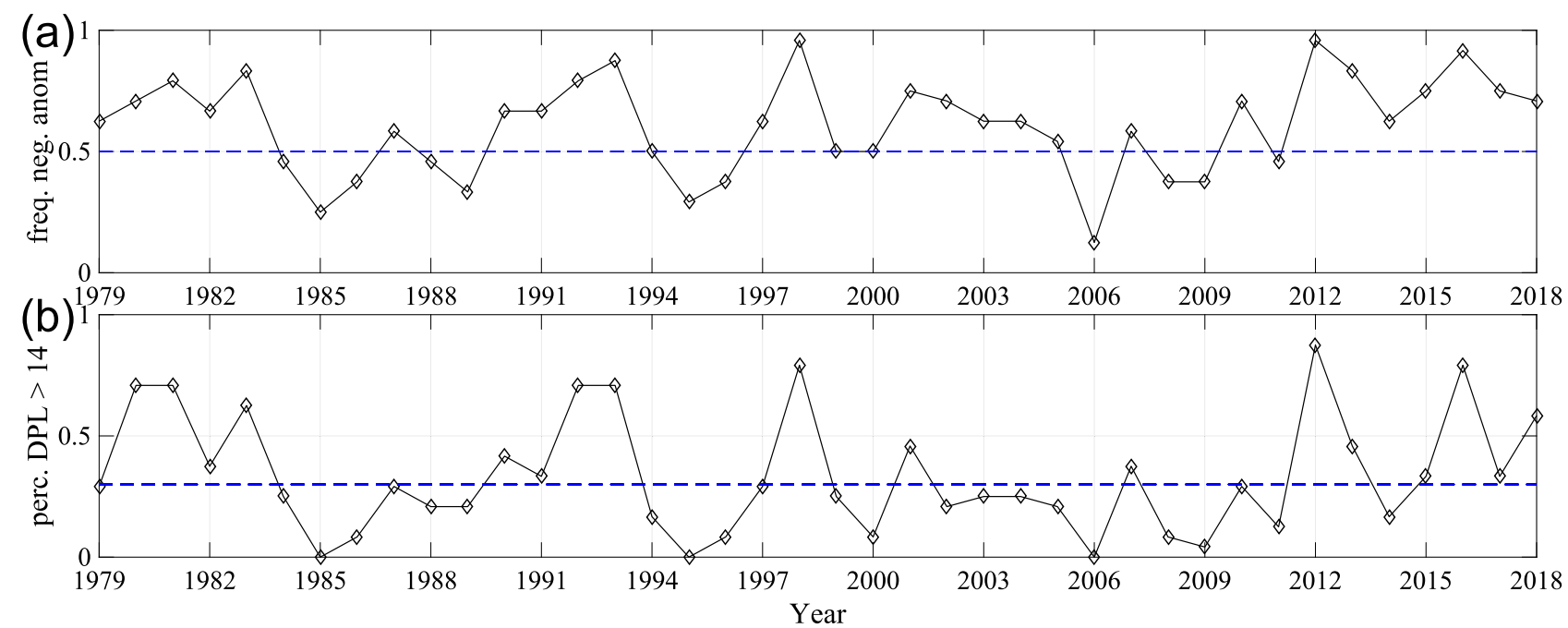

FIG. 4. (a) Frequency of negative precipitation anomaly series, and (b) the percentage of days that have drought period length longer than two weeks. 
TABLE I. Drought events as defined by DPL values larger than two weeks. Values correspond to the $i$-th $( \pm 5)$ day of each year.

\begin{tabular}{lccccccccc}
\hline \hline 2009 & 2010 & 2011 & 2012 & 2013 & 2014 & 2015 & 2016 & 2017 & 2018 \\
\hline $1-10$ & $10-15$ & $145-155$ & $1-35$ & $30-65$ & $1-35$ & $1-40$ & $35-120$ & $1-30$ & $1-25$ \\
$40-45$ & $35-45$ & $245-275$ & $55-160$ & $85-95$ & $70-75$ & $90-100$ & $150-255$ & $50-55$ & $65-85$ \\
$190-195$ & $110-120$ & $320-335$ & $185-200$ & $240-265$ & $155-165$ & $135-145$ & $285-305$ & $75-80$ & $110-200$ \\
$250-270$ & $250-280$ & $355-365$ & $235-295$ & $290-295$ & $260-265$ & $220-230$ & $355-365$ & $110-125$ & $220-235$ \\
$310-325$ & $325-330$ & & $345-365$ & & $300-305$ & $250-355$ & $260-290$ & $255-275$ \\
& & & & & $330-340$ & & $350-365$ & $350-355$ \\
& & & & & & & \\
\hline
\end{tabular}

identified for Niño 3.0 and Eastern Pacific 2010-2011 and 2018. From this perspective, the Eastern Pacific El Niño events are relatively more relevant to the drought in NEB as compared to the Central Pacific.

North Atlantic: Time lags of 2-3 months have been identified for years 2010-2011 and 2013-2016. In addition, lags of about 4 months are disclosed for 2012 and 2016. Note that
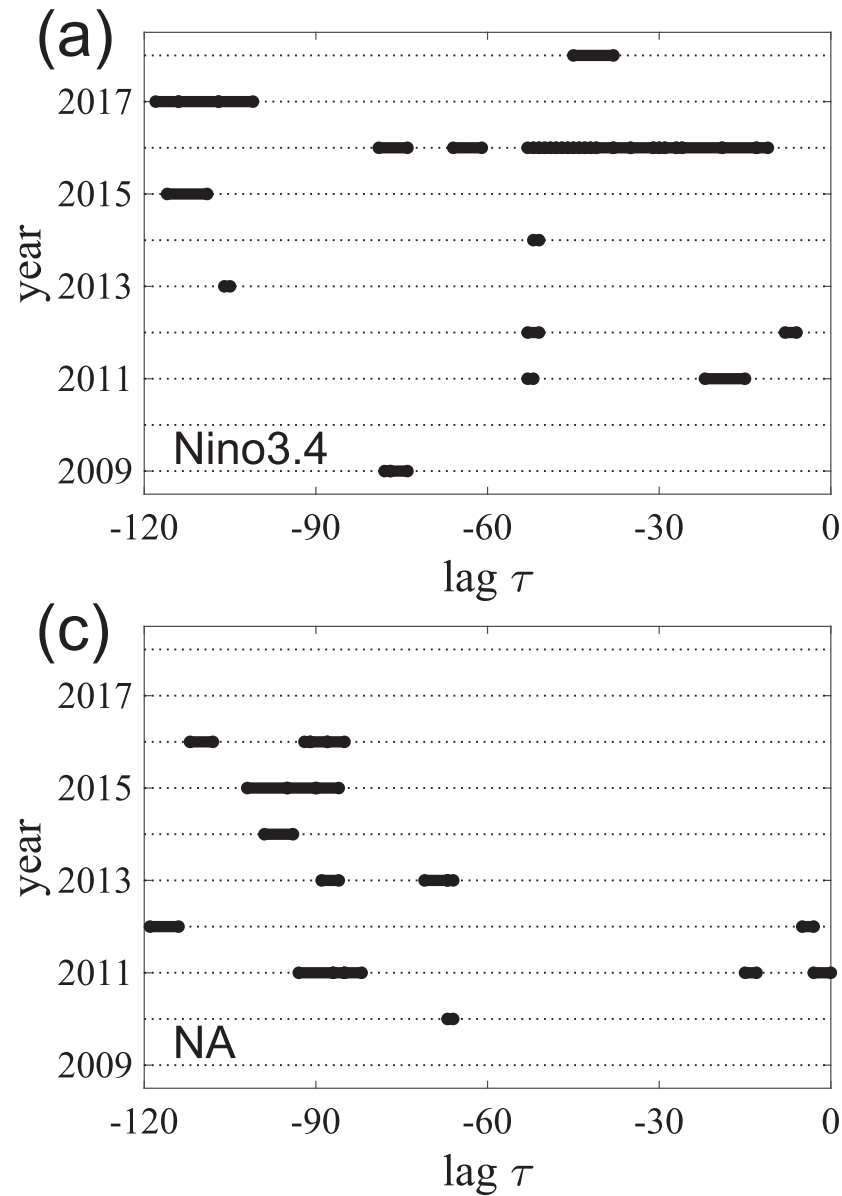

these episodic coupling delays agree with the hypothesis in the literature. ${ }^{17}$ No significant couplings exist for years 2009 and 2017-2018.

South Atlantic: Time lags of 2-3 months have been identified for years 2010-2012, 2015, and 2018. In both 2010 and 2017-2018, episodic coupling lags of about 4 months are found as well. No coupling lags are found in 2013-2014 and 2016.
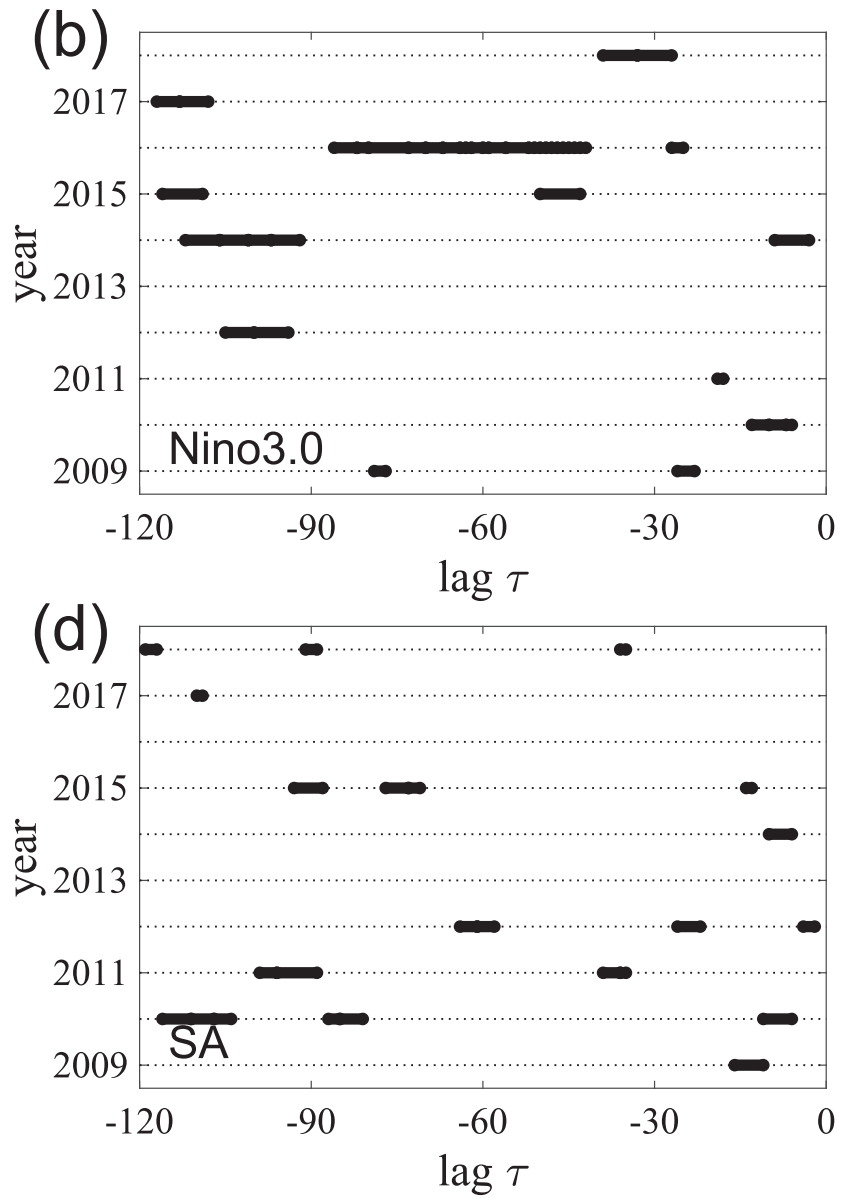

FIG. 5. Time lag influence of all ocean areas on NEB from 2009 to 2018. (a) Niño 3.4, (b) Niño 3.0, (c) North Atlantic, and (d) South Atlantic. 
TABLE II. Time lag information extracted by KLD. The symbol "/" represents cases of no significant time lags. All values correspond to days back from the 1 st January of each year.

\begin{tabular}{lcccccc}
\hline \hline Year & Niño 3.4 & Niño 3.0 & North Atlantic & South Atlantic & Central Pacific & Eastern Pacific \\
\hline 2009 & $74-78$ & $76-80$ & $/$ & $11-16$ & $73-79$ & $/$ \\
2010 & $/$ & $23-28$ & $64-67$ & $105-112,81-88$ & $77-80,23-26$ \\
2011 & $50-56,15-22$ & $17-20$ & $82-93$ & $88-99,35-40$ & $45-53,25-22$ \\
2012 & $50-57$ & $92-112$ & $114-119$ & $58-65,22-27$ & $51-53$ & $18-75$ \\
2013 & $100-108$ & $/$ & $84-89,66-72$ & $/$ & $105-106,47-51$ \\
2014 & $49-55$ & $108-116,40-50$ & $93-99$ & $/$ & $50-53$ & 100 \\
2015 & $105-116$ & $108-116$ & $86-102$ & $87-94,71-76$ & $109-116$ & $109-116,43-50$ \\
2016 & $70-79,60-67,20-55$ & $42-88$ & $107-112,85-92$ & $/$ & $11-79$ \\
2017 & $100-118$ & $108-117$ & $/$ & $109-112$ & $100-118$ \\
2018 & $38-45$ & $27-40$ & $/$ & $117-119,88-92,33-36$ & $38-45$ \\
\hline \hline
\end{tabular}

The episodic coupling lags help to define the time window of SST that needs to be shifted backward, which are used to establish the correlations to the oceans as summarized in Sec. IV C. Furthermore, the episodic coupling lags agree with the findings that have been proposed in Ref. 10, showing considerable variabilities on both spatial and temporal seasonal scales.

\section{Correlations to the oceans}

\section{General overview of SST variability}

For better comparisons, Fig. 6 shows the general overview of SST for the period 2008-2018. When studying the drought correlations in 2009, we need to shift the SST with proper coupling delays and, therefore, the SST in 2008 is included in Fig. 6. In the Pacific region, the time period when SSTs are larger than $T_{0}=0.5^{\circ} \mathrm{C}$ are highlighted, which are further used to define warmer periods in the ocean. Since the Atlantic Ocean is warmer than the Pacific, the threshold $T_{0}=0.6^{\circ} \mathrm{C}$ is chosen for the tropical Atlantic oceans. In 2009-2010, the SST in the Niño 3.4 region started warming above the threshold in 2009 and reached the maximum in December 2009 and January 2010. This El Niño event showed a relatively slow decreasing trend, which was followed by a fast warming temperature in the North and South Atlantic. In 2012, both the Pacific and NA showed some episodic but short intervals of high
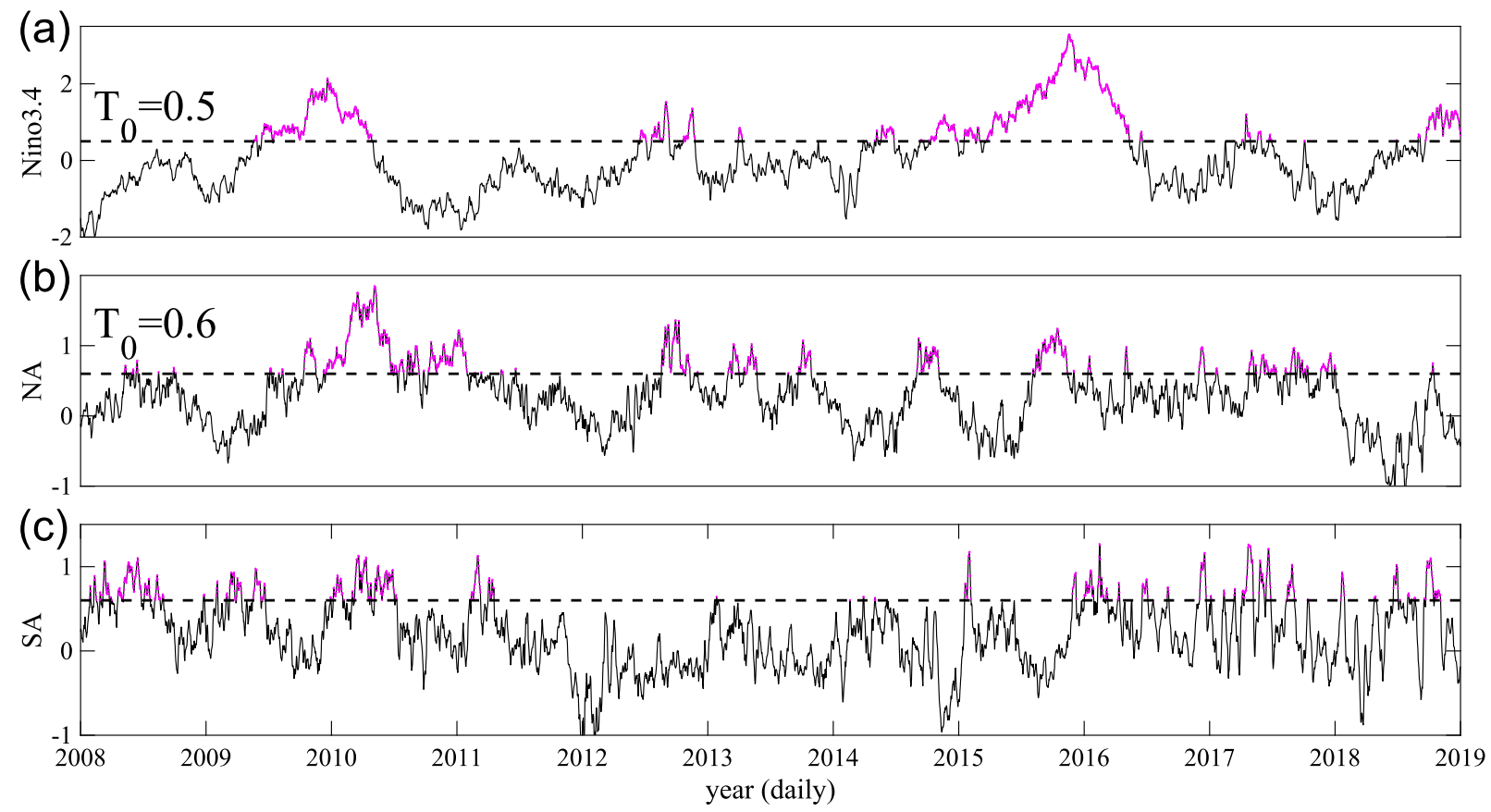

FIG. 6. SST of the surrounding ocean areas. (a) Niño 3.4, (b) North Atlantic, and (c) South Atlantic. The temporal windows of SST greater than $0.5^{\circ} \mathrm{C}$ in the Niño 3.4 (respectively, $0.6^{\circ} \mathrm{C}$ in the Atlantic) are highlighted. 
temperature [Figs. 6(a) and 6(b)]. Starting from December 2014, the Pacific showed an unprecedented warming period, which was well above the threshold for the entire 2015. This historic strong El Niño event was finally over in February 2016. During this El Niño interval, we find some time windows of high temperature in the NA and SA as well [Figs. 6(b) and 6(c)].

Figure 6 suggests an increase from 2009 to 2018 in the frequency and intensity of dry spells and droughts larger than 0.5 , which has had an intensity and impact that had not been seen in the last few decades. This result agrees with Ref. 1, which highlights that this region is under a risk of aridification, which shows that the arid areas increased throughout the NEB between 1951 and 2010.

Furthermore, Fig. 6 also reveals that driest years are recurrent in the region and as part of its natural climate variability. Previous observational studies have identified that drought events in NEB are associated with El Niño events, with strong warming in the tropical north Atlantic, or a combination of both, in general, due to an anomalously northward position of the ITCZ over the Atlantic sector. The influence from the Atlantic becomes stronger because of the warming period the multidecadal Atlantic oscillations.

\section{SST variability during extreme drought events}

In the next step, we investigate how the SSTs behave when there are drought events (DPL larger than 14 days). The coupling delays are chosen according to the previous statistical results of KLD measures, as shown in Table II. Figure 7 shows the variations of


the warming ratio [Eq. (4)] for each year. In addition, we divide the discussion into two aspects: (i) drought events in the entire period of each year [Figs. 7(a), 7(b), and 7(c)], and (ii) drought events for FMAM only [Figs. 7(d), 7(e), and 7(f)].

In 2009-2010, we find that Pacific Ocean played a stronger role first in 2009, but later in 2010 became weaker than the North Atlantic [Fig. 6(a)]. This is because an El Niño event started in the late 2009 and finished till the end of February 2010, which is followed by the anomalous warming in the NA ocean [visually shown in Figs. 6(a) and 6(b)]. The restrictions to seasons of FMAM disclose pronounced influence in 2010 from both the Pacific and NA oceans [Fig. 6(d)], which are mainly due to the overlapped time windows of high temperature in both oceans.

In 2012-2013, both the Pacific and Atlantic show warming behavior if all drought events are considered in this time period [Fig. 6(a)]. However, for the wet seasons, no warmings are found in FMAM in 2012 [Fig. 6(d)]. Therefore, the relative high ratio values in 2012 [Fig. 6(a)] are related to the dry conditions in the dry seasons. On the other hand, in 2012, we find an increased temperature gradient between the North and South Atlantic [Figs. 6(b) and $6(\mathrm{e})]$. In 2013, the relative stronger influence from the NA has been found than that from the Niño 3.4 region [Figs. 6(a) and 6(d)]. The temperature gradient between the NA and SA became weaker than 2012 [Fig. 6(b) and 6(e)], indicating a La Niña event which was relevant to the drought in the NEB. ${ }^{25}$ These results agree with model predictions, ${ }^{39}$ which show a relatively high predictability of rainfall, highlighting the important influence from the SST gradient

FIG. 7. Warming ratio of SSTs that are larger than $0.5^{\circ} \mathrm{C}$ when DPL is larger than two weeks. [(a)-(c)] for the entire year and [(d)-(f)] are for the season FMAM. [(a) and (d)] for three ocean areas of Niño 3.4, NA and SA, and [(b) and (e)] for the absolute value of the difference between the North and South Atlantic ocean. [(c) and (f)] Niño 3.4, Niño 3.0, Central and Eastern Pacific. 
between the NA and SA, though El Niño can be dominant when it is strong.

Starting from September 2014, the SST of Pacific showed an unprecedented increasing trend, reaching the maximum in October 2015 followed by an extended decreasing period until the mid of April 2016 [Fig. 6(a)]. During this extremely long period of warming in the Pacific, the NA showed two episodic warming periods September/October 2014 and the beginning of August until the end of November 2015. This unprecedented long period of the El Niño event is the main cause of the severe drought events in 2015-2016, which was further influenced by the episodic increasing of the temperature gradient between the NA and SA.

All the above results in related to the influence from the Pacific have been consistently obtained from the Niño 3.0, Central and Eastern Pacific [Figs. 6(c) and 6(f)]. Furthermore, the identified nonstationary coupling directions from the oceans agree with the results that have been published in the literature. ${ }^{6,7,10,13,40}$

\section{DISCUSSIONS}

Based on ordinal partition transition network approaches for nonlinear time series analysis, we propose an algorithm to establish the episodic coupling directions on intraseasonal time scales from the ocean to the extreme precipitation patterns in the northeast Brazil. This method involves three steps: (i) defining drought events by computing drought period length, (ii) statistically identifying coupling lags from the ocean to the precipitation patterns, and (iii) calculating warming ratios of the SST of the surrounding oceans during time windows of drought events with proper time lags. We note that the computation of DPL captures the intraseasonal temporal properties of the precipitation anomalies, which can be applied for other purposes of extreme value analysis. Furthermore, the complexity Kullback-Leibler divergence measure is shown to be useful to estimate statistically significant coupling directions. In the particular case of this study, our method shows capabilities in detecting the coupling delays whenever the SST shows warming periods above the thresholds, namely, the SST anomaly is larger than $0.5^{\circ} \mathrm{C}$ in the Pacific while $0.6^{\circ} \mathrm{C}$ in the Atlantic oceans.

We note that time lags of the directional coupling from the oceans vary considerably from year to year. On average, time lags of 2-3 months have been identified from the ocean regions. However, the Pacific showed an increased lag up to 4 months for years 2015 and 2017. In other years, one month lag is identified in 2010-2011 and 2018, especially for Niño 3.0 and Eastern Pacific since these two regions are closer to the South America. We have obtained consistent results when considering different ENSO types of the Pacific areas because we focused on a relatively narrow area in the NEB, instead of a wider region of South America. ${ }^{26-28,41}$ Similarly, the Atlantic Ocean has an average time lag of 2-3 months. Lags of 4 months are found for 2012 and 2016 in the North Atlantic, and in 2010 and 2017 in the South Atlantic. Our results of coupling delays on intraseasonal time scales are consistent with the results in Refs. 10 and 42 , as the interactions often show considerable episodic time scales ranging from seasonal to interannual scales. Therefore, from the viewpoint of the individual event, the coupling lags as shown in Fig. 5 and Table II are helpful for understanding the specific feature for individual drought.
From the viewpoint of methodological perspective, the OPTN time series network approach identifies intraseasonal scale properties that are potential for monitoring and forecasting droughts in the NEB, in order to propose effective strategies for drought risk reduction. Furthermore, the Kullback-Leibler divergence measure is based on ordinal patterns of time series, which does not require a presumption of stationarity of time series. The statistical significance of the results is easily assessed by either phase randomization ${ }^{36}$ or by order pattern transition randomization. Our method is useful to establish coupling directions in rapidly changing time scales that often require a considerably longer time span of the predictions. ${ }^{43}$ Therefore, the intraseasonal directional coupling delays identified by the KLD measures show high potential for regional climate model predictions for the climate conditions.

Further investigation is necessary to better understand the effects of the warming phase for the multidecadal north-south Atlantic oscillation on the precipitation patterns in the NEB. ${ }^{44}$ This becomes crucial for the entire tropical Atlantic basin, most apparent in the north Atlantic tropical region, warmed up since mid-1970s. ${ }^{40}$ It remains to be a challenge since an indirect effect of the ENSO on the SST variabilities in the tropical Atlantic exists, ${ }^{7}$ which requires to consider the possible indirect effects. On the other hand, MJO from the Pacific should also be studied since the SST from the western Pacific may influence the intraseasonal precipitation variability in the South America. ${ }^{15}$ The question of considering indirect effects of mode interaction on precipitation cannot be fully answered by our proposed network method yet, which requires further investigations.

\section{ACKNOWLEDGMENTS}

Parts of this work were financially supported by the National Natural Science Foundation of China (Grant Nos. 11872182 and 11835005), the DFG/FAPESP (Grant Nos. IRTG 1740/TRP 2011/50151-0 and 2015/50122-0), and the National Institute of Science and Technology for Climate Change Phase 2 under CNPq grant (No. 465501/2014-1), FAPESP grant, and the National Coordination for High Level Education and Training (CAPES) grant (No. 88887.136402-00INCT). Y. Z. further acknowledges the support by Shanghai Municipal Science and Technology Major Project (No. 2018SHZDZX01) and ZJ Lab.

\section{DATA AVAILABILITY}

The data that support the findings of this study are openly available. CPC Global Unified Precipitation and NOAA High Resolution SST data are provided by the NOAA/OAR/ESRL PSD, Boulder, CO, USA, from their Web site at https://www.esrl.noaa.gov/psd/, Refs. 22-24.

\section{REFERENCES}

${ }^{1}$ J. A. Marengo, R. R. Torres, and L. M. Alves, "Drought in northeast Brazil-Past, present, and future," Theor. Appl. Climatol. 129(3), 1189-1200 (2017).

${ }^{2}$ J. A. Marengo, A. P. Cunha, W. R. Soares, R. R. Torres, L. M. Alves, S. S. de Barros Brito, L. A. Cuartas, K. Leal, G. R. Neto, R. C. S. Alvalá, and A. R. Magalhaes, Increase Risk of Drought in the Semiarid Lands of Northeast Brazil Due to Regional Warming Above $4^{\circ} \mathrm{C}$ (Springer, Cham, 2019). pp. 181-200. 
${ }^{3}$ R. C. S. Alvalá, A. P. M. A. Cunha, S. S. B. Brito, M. E. Seluchi, J. A. Marengo, O. L. L. Moraes, and M. A. Carvalho, "Drought monitoring in the Brazilian semiarid region,” An. Acad. Bras. Cienc. 91(Suppl. 1), e20170209 (2019).

${ }^{4}$ S. Hastenrath and L. Heller, "Dynamics of climatic hazards in northeast Brazil," Quart. J. Roy. Meteor. Soc. 103(435), 77-92 (1977).

${ }^{5}$ R. R. Rodrigues and M. J. McPhaden, "Why did the 2011-2012 La Niña cause a severe drought in the Brazilian Northeast?,” Geophys. Res. Lett. 41(3), 1012-1018, (2014).

${ }^{6}$ A. D. Moura and J. Shukla, "On the dynamics of droughts in Northeast Brazil: Observations, theory and numerical experiments with a general circulation model,” J. Atmos. Sci. 38, 2653-2675 (1981).

${ }^{7}$ A. Giannini, J. C. H. Chiang, M. A. Cane, Y. Kushnir, and R. Seager, "The ENSO teleconnection to the tropical Atlantic ocean: Contributions of the remote and local SSTs to rainfall variability in the tropical Americas," J. Clim. 14(24), 4530-4544 (2001).

${ }^{8}$ A. Timmermann, S.-I. An, J.-S. Kug, F.-F. Jin, W. Cai et al., "El Niño southern oscillation complexity," Nature 559(7715), 535-545 (2018).

${ }^{9}$ R. Kane, "Prediction of droughts in North-East Brazil: Role of ENSO and use of periodicities," Int. J. Climatol. 17, 655-665 (1997).

${ }^{10}$ C. B. Uvo, C. A. Repelli, S. E. Zebiak, and Y. Kushnir, "The relationships between Tropical Pacific and Atlantic SST and Northeast Brazil monthly precipitation," J. Clim. 11(4), 551-562 (1998).

${ }^{11}$ R. V. Andreoli and M. T. Kayano, "Tropical Pacific and South Atlantic effects on rainfall variability over northeastern Brazil,” Int. J. Climatol. 26, 1895-912 (2006).

${ }^{12}$ M. T. Kayano, R. V. Andreoli, and R. A. F. Souza, "Evolving anomalous SST patterns leading to ENSO extremes: Relations between the tropical Pacific and Atlantic Oceans and the influence on the South American rainfall," Int. J Climatol. 31, 1119-1134 (2011).

${ }^{13}$ C. Wang, ENSO, Atlantic Climate Variability and the Walker and Hadley Circulations (Springer, Dordrecht, 2004), pp. 173-202.

${ }^{14} \mathrm{M}$. T. Kayano and R. V. Andreoli, "Relationships between rainfall anomalies over northeastern brazil and the El Niño-southern oscillation,” J. Geophys. Res. 111, D13101, (2006)

${ }^{15}$ R. V. Andreoli and M. T. Kayano, "ENSO-related rainfall anomalies in South America and associated circulation features during warm and cold Pacific decadal oscillation regimes,” Int. J. Climatol. 25, 2017-2030 (2005).

${ }^{16}$ J. Runge, V. Petoukhov, and J. Kurths, "Quantifying the strength and delay of climatic interactions: The ambiguities of cross correlation and a novel measure based on graphical models," J. Clim. 27(2), 720-739 (2014).

${ }^{17}$ A. M. T. Ramos, Y. Zou, G. Sampaio, J. Kurths, and E. E. N. Macau, "Unveiling non-stationary coupling between Amazon and ocean during recent extreme events," Clim. Dyn. 50, 767-776 (2018).

${ }^{18}$ J. Runge, V. Petoukhov, J. F. Donges, J. Hlinka, N. Jajcay, M. Vejmelka, D. Hartman, N. Marwan, M. Palus, and J. Kurths, "Identifying causal gateways and mediators in complex spatio-temporal systems," Nat. Commun. 6, 8502 (2015).

${ }^{19}$ N. Boers, B. Goswami, A. Rheinwalt, B. Bookhagen, B. Hoskins, and J. Kurths, "Complex networks reveal global pattern of extreme-rainfall teleconnections," Nature 566, 373-377 (2019).

${ }^{20} \mathrm{M}$. Wiedermann, A. Radebach, J. F. Donges, J. Kurths, and R. V. Donner, "A climate network-based index to discriminate different types of El Niño and La Niña," Geophys. Res. Lett. 43, 7176-7185, (2016).

${ }^{21}$ Y. Zou, R. V. Donner, N. Marwan, J. F. Donges, and J. Kurths, "Complex network approaches to nonlinear time series analysis," Phys. Rep. 787, 1-97 (2019).

${ }^{22}$ M. Chen, W. Shi, P. Xie, V. B. S. Silva, V. E. Kousky, R. W. Higgins, and J. E. Janowiak, "Assessing objective techniques for gauge-based analyses of global daily precipitation,” J. Geophys. Res. 113, D04110 (2008).

${ }^{23}$ P. Xie, A. Yatagai, M. Chen, T. Hayasaka, Y. Fukushima, C. Liu, and S. Yang, "A gauge-based analysis of daily precipitation over east asia," J. Hydrometeor. 8(3), 607-626 (2007).
${ }^{24}$ R. W. Reynolds, T. M. Smith, C. Liu, D. B. Chelton, K. S. Casey, and M. G. Schlax, "Daily high-resolution-blended analyses for sea surface temperature," J. Clim. 20, 5473-5496 (2007).

${ }^{25}$ M. T. Kayano, R. V. Andreoli, and R. A. F. de Souza, "How the two nodes of the tropical atlantic sea surface temperature dipole relate the climate of the surrounding regions during austral autumn," Int. J. Climatol. 38(8), 3927-3941 (2018).

${ }^{26}$ R. G. Tedeschi, A. M. Grimm, and I. F. A. Cavalcanti, "Influence of Ccentral and east ENSO on extreme events of precipitation in south america during austral spring and summer," Int. J. Climatol. 35(8), 2045-2064 (2015).

${ }^{27}$ R. G. Tedeschi, A. M. Grimm, and I. F. A. Cavalcanti, "Influence of central and east ENSO on precipitation and its extreme events in south america during austral autumn and winter," Int. J. Climatol. 36, 4797-4814 (2016).

${ }^{28}$ R. V. Andreoli, S. S. de Oliveira, M. T. Kayano, J. Viegas, R. A. F. de Souza, and L. A. Candido, "The influence of different El Niño types on the South American rainfall,” Int. J. Climatol. 37(3), 1374-1390 (2017).

${ }^{29}$ Y. Zou, E. E. N. Macau, G. Sampaio, A. M. T. Ramos, and J. Kurths, "Do the recent severe droughts in the Amazonia have the same period of length?," Clim. Dyn. 46, 3279-3285 (2016).

${ }^{30}$ Y. Zou, E. E. N. Macau, G. Sampaio, A. M. T. Ramos, and J. Kurths, "Characterizing the exceptional 2014 drought event in São Paulo by drought period length," Clim. Dyn. 51, 433-442 (2018).

${ }^{31}$ M. McCullough, M. Small, T. Stemler, and H. H.-H. Iu, "Time lagged ordinal partition networks for capturing dynamics of continuous dynamical systems," Chaos 25(5), 053101 (2015).

${ }^{32}$ M. McCullough, M. Small, H. H. C. Iu, and T. Stemler, "Multiscale ordinal network analysis of human cardiac dynamics," Philos. Trans. R. Soc. A Math. Phys. Eng. Sci. 375(2096), 20160292 (2017).

${ }^{33}$ Y. Ruan, R. V. Donner, S. Guan, and Y. Zou, "Ordinal partition transition network based complexity measures for inferring coupling direction and delay from time series," Chaos 29(4), 043111 (2019).

${ }^{34} \mathrm{C}$. Bandt and B. Pompe, "Permutation entropy: A natural complexity measure for time series,” Phys. Rev. Lett. 88(17), 174102 (2002).

${ }^{35}$ J. Amigó, Permutation Complexity in Dynamical Systems (Spinger, Berlin/ Heidelberg, 2010).

${ }^{36}$ J. Theiler, S. Eubank, A. Longtin, B. Galdrikian, and J. Doyne Farmer, "Testing for nonlinearity in time series: The method of surrogate data," Phys. D 58(1-4), 77-94 (1992).

${ }^{37}$ K. E. Trenberth, “The definition of El Niño," Bull. Am. Meteor. Soc. 78, 2771-2777 (1997).

${ }^{38}$ S. S. B. Brito, A. P. M. A. Cunha, C. C. Cunningham, R. C. Alvalá, J. A. Marengo, and M. A. Carvalho, "Frequency, duration and severity of drought in the semiarid northeast brazil region," Int. J. Climatol, 38(2), 517-529 (2018).

${ }^{39}$ C. K. Folland, A. W. Colman, D. P. Rowell, and M. K. Davey, "Predictability of northeast brazil rainfall and real-time forecast skill, 1987-98,” J. Clim. 14(9), 1937-1958 (2001)

${ }^{40}$ J. Servain, G. Caniaux, Y. K. Kouadio, M. J. McPhaden, and M. Araujo, "Recent climatic trends in the tropical atlantic," Clim. Dyn. 43(11), 3071-3089 (2014).

${ }^{41}$ H.-Y. Kao and J.-Y. Yu, "Contrasting Eastern-Pacific and Central-Pacific types of ENSO,” J. Clim. 22(3), 615-632 (2009).

${ }^{42} \mathrm{~V}$. Torralba, B. Rodriguez-Fonseca, E. Mohino, and T. Losada, "The nonstationary influence of the Atlantic and Pacific Niños on North Eastern South American rainfall," Front. Earth Sci. 3, 55 (2015).

${ }^{43}$ P. Nobre, J. A. Marengo, I. F. A. Cavalcanti, G. Obregon, V. Barros, I. Camilloni, N. Campos, and A. G. Ferreira, "Seasonal-to-decadal predictability and prediction of South American climate," J. Clim. 19(23), 5988-6004 (2006).

${ }^{44}$ M. T. Kayano and V. B. Capistrano, "How the Atlantic multidecadal oscillation (AMO) modifies the ENSO influence on the South American rainfall," Int. J. Climatol. 34(1), 162-178 (2014). 